\title{
Initial Statistics from the Perth Automated Supernova Search
}

\author{
Andrew J. Williams \\ Perth Observatory, Department of Conservation and Land Management, \\ Walnut Rd, Bickley, WA 6076, Australia \\ andrew@pd.uwa.edu.au \\ Received 1996 December 19, accepted 1997 April 27
}

\begin{abstract}
The Perth Automated Supernova Search uses the 61-cm PLAT (Perth Lowell Automated Telescope) at Perth Observatory, Western Australia. Since 1993 January 1, five confirmed supernovae have been found by the search. The analysis of the first three years of data is discussed, and preliminary results presented. We find a Type $\mathrm{Ib} / \mathrm{c}$ rate of $0.43 \pm 0.43 \mathrm{SNu}$, and a Type IIP rate of $0.86 \pm 0.49 \mathrm{SNu}$, where $\mathrm{SNu}$ are 'supernova units', expressed in supernovae per $10^{10} \mathrm{~L}_{\mathrm{B}_{\odot}}$ galaxy per century. These values are for a Hubble constant of $75 \mathrm{kms}^{-1} \mathrm{Mpc}^{-1}$, and scale as $\left(H_{0} / 75\right)^{2}$.
\end{abstract}

Keywords: stars: statistics - supernovae: general

\section{Introduction}

The Perth Automated Supernova Search has been running since late 1992, and has found five confirmed supernovae (SNe) to date. It was organised and funded by the Perth Astronomy Research Group (PARG), consisting of members from four Perth universities and Perth Observatory. The Perth/Lowell 61-cm Boller and Chivens telescope at Perth Observatory was fitted with computer controlled stepper motors to allow automation, and a liquid $\mathrm{N}_{2}$ cooled $\mathrm{CCD}$ camera based on a Palomar design (Gunn et al. 1987) was built for the search. A complete description of the search hardware and software has been given elsewhere (Williams et al. 1995). The search began operating late in 1992, and the period analysed here is the three years from 1993 January 1 to 1995 December 31.

The aim of the Perth search was not just to find as many $\mathrm{SNe}$ as possible, but to try to establish a system able to obtain supernova rates with less dependence on poorly known parameters than other existing rate determinations. The strategy has been to look at nearby galaxies and to repeat observations frequently, with observations generally separated by only a few days. Our galaxies were selected from 'The ESO/Uppsala Survey of the ESO(B) Atlas' on the ADC CD-ROM. To maximise the number of supernovae found, we chose to observe only late spiral galaxies (Sbc-Sd), because other SN searches have found that these galaxies produce more supernovae (Tammann, Löffler \& Schröder 1994). While this is an assumption based on existing SN statistics, it will not bias our final result, which is an independent estimate for $\mathrm{SN}$ rates in late spiral galaxies.
Taking frequent observations also leads to early discoveries, an important goal in itself. The hardware available at Perth is obviously not suitable for finding the high-redshift supernovae most valuable as distance estimators. Discovering a smaller number of supernovae near maximum (where they have intrinsic scientific value beyond their inclusion in the statistics) was thought to be better than finding a larger number of medium-distance supernovae weeks or months after maximum. It would also give a rate estimate with less dependence on light curves and other poorly known parameters.

The CCD camera built for the search has a $576 \times 384$ pixel field $\left(5 \cdot 6^{\prime} \times 3 \cdot 7^{\prime}\right.$ arc field, $0 \cdot 58^{\prime \prime}$ pixels), and was used unfiltered, with 180 -second exposures, giving an estimated detection limit of about $16.5 \mathrm{mag}$ for most of the three-year search period. Changes in the electronics improved this to 17 mag over the last 12 months analysed.

Lack of resources meant that a fully unattended search was impractical - there is no weather monitoring system or back-up power supply, for example. Although the search itself is fully automatic, one person needed to be on site to fill the $\mathrm{N}_{2}$ dewar at the start of the night, and monitor the weather. The search runs as a background project, using available telescope and operator time. Over the three years analysed here, we acquired 5540 search images, representing around 370 hours of telescope time, plus overhead each night for flatfielding, etc.

\section{Supernovae}

Supernovae were originally classified into two spectroscopic classes. These were the 'Type I' events that showed no evidence of hydrogen in their 
spectra, and the 'Type II' events that did show strong hydrogen lines. SNe I were found in all galaxy types - including those without evidence for recent star formation - and therefore had old, highly evolved progenitors. SNe II occurred only in star-forming galaxies, and presumably had young, massive progenitors.

The current classification system for $\mathrm{SNe}$ is rather more complex. The Type I class has been split into $\mathrm{Ia}, \mathrm{Ib}$ and Ic events, based on the presence of $\mathrm{Si}$ II and He I lines in the spectra, and the Type II class has been split into IIP ('Plateau' ) and IIL ('Linear' ) events based on the light curve shape. Table 1 gives a summary of supernova classes.

Table 1. Supernova types in order of increasing envelope thickness

\begin{tabular}{|c|c|c|}
\hline Type & Spectral features & Probable mechanism \\
\hline Ia & $\begin{array}{l}\text { Si II } \\
\text { No H, He }\end{array}$ & $\begin{array}{l}\text { White dwarf accretion } \\
\text { or coalescence }\end{array}$ \\
\hline Ic & No $\mathrm{H}, \mathrm{He}$, or $\mathrm{Si}$ & $\begin{array}{l}\text { Core collapse in } \\
\text { massive star with no } \mathrm{H} \\
\text { and thin or missing } \mathrm{He} \\
\text { envelope. }\end{array}$ \\
\hline $\mathrm{Ib}$ & $\begin{array}{l}\text { He I } \\
\text { No } \mathrm{H} \text { or } \mathrm{Si}\end{array}$ & $\begin{array}{l}\text { Core collapse in } \\
\text { massive star with no } \mathrm{H} \\
\text { envelope }\end{array}$ \\
\hline IIL & $\mathrm{H}$ & $\begin{array}{l}\text { Core collapse in } \\
\text { massive star with thin } \\
\mathrm{H} \text { envelope }\end{array}$ \\
\hline IIP & $\begin{array}{l}\text { H, with } \\
\text { P-Cygni profile }\end{array}$ & $\begin{array}{l}\text { Core collapse in } \\
\text { massive star }\end{array}$ \\
\hline
\end{tabular}

Type Ia events are believed to be thermonuclear explosions of $\mathrm{C}-\mathrm{O}$ white dwarfs, caused either by a coalescence of a binary pair of white dwarfs, or accretion onto a white dwarf from a giant companion. The Type Ia are believed to make good 'standard candles' and have very uniform properties, with only a few easily recognised exceptions (Branch et al. 1995).

The rest of the SN types (Ib, Ic, IIP and IIL) are apparently core-collapse events in massive stars. The core exceeds the Chandrasekhar limit and collapses to a neutron star or black hole. The rest of the stellar material initially falls in, but 'bounces', forming a shockwave that, unassisted, would run out of energy before reaching the stellar surface. The shockwave is prevented from stalling by heating from neutrinos emitted by electron capture in the core, and the re-heated shockwave tears the star apart.

For a core-collapse event, after shock breakout and initial rapid cooling, the light curve and spectrum is dominated by the outer envelope-a thick hydrogen envelope results in an extended plateau in the light curve (a Type IIP) as the hydrogen shell expands outwards (increasing the black-body emission radius) and cools (becoming transparent and moving the photosphere inwards through the ejected envelope). If these two effects balance, there is a long period of essentially constant luminosity. When the photosphere reaches the inner regions where ${ }^{56} \mathrm{Ni}$ and ${ }^{56} \mathrm{Co}$ generated in the explosion are mixed, there is an extension of the plateau, or even a secondary maximum, as the radioactive decay heats the material. After this, the light curve shape is an exponential tail from the ${ }^{56} \mathrm{Ni}-{ }^{56} \mathrm{Co}-{ }^{56} \mathrm{Fe}$ decay, modified by changes in the transparency, as the capture efficiency for the gamma photons from the decay is reduced (Eastman et al. 1994; Blanton et al. 1995).

The other SN types (IIL, Ib and Ic) are thought to result from core-collapse in stars that have lost all or most of their envelopes. The IIL events are $\mathrm{SNe}$ where the $\mathrm{H}$ shell is fairly thin, not leading to a light curve plateau. The Ib and Ic events are from stars where all or most of the $\mathrm{H}$ and $\mathrm{H} / \mathrm{He}$ envelopes respectively are missing, either from normal mass-loss in massive stars or from Roche-lobe overflow in a binary system (Woosley, Langer \& Weaver 1993, 1995).

There is a good chance that some or all of the core-collapse SN types form a continuum rather than distinct classes, as the envelope thickness of the progenitor is varied. Several supernovae have been observed to change their spectral type as they evolved (SN1987K, for example, changed from a Type II to a Type Ic spectrum, probably indicating a very thin envelope). Some intermediary SNe have been observed, and given a new class, IIb (e.g. SN1993J) - they seem to have a combination of the II and Ib properties. There is also another class of objects (IIn) that seem to be $\mathrm{SNe}$ embedded in a massive stellar outflow, presumably occurring in stars that are losing mass rapidly. Their light curves decline very slowly.

Classification of supernovae has changed drastically, so existing databases of $\mathrm{SNe}$, and estimates of $\mathrm{SN}$ rates, must be treated cautiously. Older supernovae classified as 'Type I' could have been $\mathrm{Ia}, \mathrm{Ib}$ or Ic events. Later SNe classified as 'Type Ib' could have been either Ib or Ic events. These events are often described as 'Type Ibc' or 'Type Ib/c' because of the similarity between the two. Also, differences between IIP and IIL spectra have only recently been recognised, and are often not definitive, so most are simply described as 'Type II' unless long-term light curve photometry has been taken.

\section{Control Time}

To arrive at a 'supernova rate', there are three essential elements:

(1) A set of observations of galaxies, each searched for supernovae.

(2) An estimate of how much observation time each galaxy has accumulated over the whole search.

(3) Some SNe discovered by the search. 
Determining item (2) accurately is crucialunfortunately, because supernovae vary so much in light curve shape and peak luminosity, this 'Control Time' must be calculated separately for each type of supernova. For example, suppose that a given galaxy is at a distance such that SNe IIP are visible for 100 days, but $\mathrm{SNe} \mathrm{Ib} / \mathrm{c}$ only for 10 days. One year of observations of that galaxy taken once per month would find all of the IIP events, but only around one third of the $\mathrm{Ib} / \mathrm{c}$ events, as there would be about 20 days per month in which a Ib/c supernova could have occurred, then faded below the detection threshold before the next observation.

We can quantify this by defining a control time for each observation, specific to each type of supernova. The control time for an observation is defined as either the time since the previous observation of that galaxy (1), or the time a supernova of a given type would have been detectable in that galaxy (2), whichever is less.

If we follow this through for the above example, the IIP control time for each observation is one month, since the first clause applies (apart from the first observation). The Ib/c control time for each observation is only 10 days, since the second clause applies. This means that our one-year period of thirteen observations has found all the SNe IIP in 460 days ( 100 plus $12 \times 30$ ) in that galaxy, and all the $\mathrm{SNe} \mathrm{Ib} / \mathrm{c}$ in 130 days $(13 \times 10)$ in the same galaxy. The first observation must be treated carefully, as the absence of an object present in earlier observations might not be detected as a supernova, and this can have a significant effect on the final control time.

Being able to calculate a detection threshold for each type of supernova means knowing:

- The peak absolute magnitude and light curve shape.

- The distance and extinction to each search galaxy.

- A consistent limiting detection magnitude for the search system.

In practice, of course, none of these can be determined precisely. Apart from the standard SNe Ia, supernova light curves and luminosities vary widely, even within a class, and there could well exist a continuum of supernova types instead of the existing discrete classifications. Galaxy distances and extinctions are difficult to pin down, except for a small sample of galaxies. Finally, any detection system has to cope with widely varying sky conditions, and it is difficult to quantify how the detection efficiency changes with varying SN location within the galaxy and varying galaxy inclination angles. Visual, CCD, and photographic searches have very different dynamic range, sensitivity, and detection efficiency.

Almost all of the SN type splitting started out with one or two 'anomalous' low luminosity events that later turned out to be fortuitously discovered samples of a new type, as common (or more common) than the original. Using existing SN statistics to calculate new data can lead to circular logic. For example, early supernova searches assumed that all type I SNe were equally bright and visible for long periods, so galaxy observations were widely spaced. This led to discovery of far more of the high luminosity SNe Ia than other (then indistinguishable) class I subtypes. The result was a final 'Type I' rate estimate that was approximately that of the bright, Type Ia events, instead of the sum of all of the Type I subclass rates.

It is clear that to make a final estimate of control time (and hence supernova rates) as accurate as possible, we must try to keep the interval between observations small compared to the estimated SN detection time, so that the control time for each observation does not depend on poorly known parameters such as light curve shape and detection sensitivity.

We used four light-curve templates for the analysis-Ia, Ib/c, IIP and IIL, simplified versions of those used by Richmond (1992). The light curve templates used are shown in Figure 1, superimposed on a scatter diagram of observation intervals. Each dot represents one observation - its position on the horizontal axis is the number of days since the last observation of that galaxy, and its height denotes the absolute magnitude of the faintest SN detectable in that observation. If a point is below a given light-curve template, then all SNe of that type would have been detectable in the time since the last image of that galaxy - if not, then there is some period of time just after the last observation in which a SN of that type could have occurred, then faded below the detection limit. (Another figure-not reproduced here as it requires colour-gives much the same information in an alternate form. It can be seen in the online version of this paper on the Electronic PASA web site: http://www.atnf.csiro.au/pasa.)

Table 2. Supernova discoveries by the Perth search

\begin{tabular}{lll}
\hline Name & Type & Estimated age \\
\hline SN1993K & IIP & Near maximum \\
SN1994R & IIP & 2 weeks after maximum \\
SN1994AI & Ic & Before maximum \\
$3 / 8 / 1995$ & $?$ & Unconfirmed \\
SN1995W & IIP & 4 weeks after maximum \\
SN1996N & Ib/c & 2 weeks after maximum \\
\hline
\end{tabular}

\section{Results}

Since it began, the Perth search has discovered six supernovae, five of which have been confirmed (Williams \& Martin 1993, 1994, 1995, 1996; Martin \& Williams 1994). The unconfirmed event was almost certainly a supernova, and was re-observed locally over several nights, but was very close to 





the Sun (setting just after twilight) and was not confirmed independently. Five of these events were discovered over the three years analysed here (1993 January to 1995 December). The list of discoveries to date is shown in Table 2.

The SNe II have been taken as Type IIP supernovae on the basis of our follow-up photometry (SN1993K) or P-Cygni profiles reported in the spectra taken to confirm the object (SN1994R and SN1995W). For the purposes of our statistics, we have allocated the unconfirmed event a $39 \%$ weight as a Type IIP and a $10 \%$ weight as a Type $\mathrm{Ib} / \mathrm{c}$, based on relative rates determined by the Asiago supernova search (Cappellaro et al. 1993).

Figure 1 shows the supernovae discovered in the three-year analysis period superimposed on the scatter plot of observation intervals, with the vertical coordinate giving the detection threshold in that galaxy, not the observed SN magnitude. The 'error' bars from SN1994R and SN1995W indicate the estimated dates that these events were at maximum light.

Another event, supernova SN1995AD, was discovered elsewhere late in 1995 (Evans 1995), in one of the galaxies in our search list. We were not observing that galaxy at the time of the event, so it does not affect these statistics. Early in 1996, after the search period analysed here, our system did independently 'discover' this supernova, and it will be included in the analysis of the 1996 search data.

It has been proposed that galaxies close to face on (inclination angle $<30^{\circ}$ ) show a greatly increased supernova frequency. The Berkeley search, using equipment and techniques similar to ours found no evidence for this effect (Muller et al. 1992), and a chi-square test on our data set also rejected an inclination dependence. We have assumed it is an effect limited to photographic searches, and no corrections were made for possible inclination effects in our data. We have, however, normalised each galaxy's control time using its integrated B magnitude, since SN frequency has been found to be proportional to the blue luminosity of the parent galaxy (Cappellaro et al. 1993; Tammann et al. 1994).

Table 3. Normalised total control times (days)

\begin{tabular}{lcc}
\hline Type & Control time & Threshold error \\
\hline Type Ia & 384 & $+44 /-44$ \\
Type Ib/c & 253 & $+58 /-50$ \\
Type IIP & 396 & $+76 /-68$ \\
Type IIL & 147 & $+64 /-48$ \\
\hline
\end{tabular}

Control times for each observation were summed, normalised to a galaxy luminosity of $10^{10} \mathrm{~L}_{\mathrm{B}_{\odot}}$ (using $\mathrm{M}_{\mathrm{B}_{\odot}}=5 \cdot 48$ ). Total control times for the three years of search data are given in Table 3 . The 'threshold errors' given were found by setting the detection limits $0.5 \mathrm{mag}$ higher and lower, and finding the new control times. These threshold errors are an estimate of the combined effect of light curve errors, galaxy distance errors, and unknown variations in the detection threshold for the system, and are only a reasonable guess for the actual errors in the control times.

Despite the fact, clearly shown in Figure 1, that the majority of observations would have detected all SNe since the last image of that field, the Type IIL control time is still less than half that of the Ia and IIP events. This is because the controltime contribution from the inter-season 'gaps' in the observations of each galaxy is large for the IIP and Ia events, and much smaller for the IIL events. There are only a few points in Figure 1 at large intervals, but each contributes greatly to the total control time. This also means that the IIL control times have more dependence on light-curve and detection threshold errors than the IIP and Ia events. Changing the detection threshold by 0.5 mag, for example, alters the control times (and hence the rates) by about $12 \%$ for Ia, $21 \%$ for Ibc, $18 \%$ for IIP and $40 \%$ for IIL events.

Figure 1-Days between search images. This plot shows every observation taken in the three-year search period as a small dot, with the horizontal (time) axis 'folded' to indicate the time, in days, between that observation and the previous image of that galaxy. The vertical axis shows the detection limit, as an absolute $\mathrm{R}$ magnitude cut-off for supernovae in that galaxy. To show how useful each observation was at detecting supernovae, the absolute R light curves of the various supernova types are superimposed, for hypothetical 'worst case' supernovae occurring immediately after the last observation of the field (scaled to $H_{0}=75 \mathrm{~km} \mathrm{~s}^{-1} \mathrm{Mpc}^{-1}$ ).

For example, a point in the bottom left represents an image taken very soon after a previous observation, and of a galaxy so close that any supernovae since the last observation will be many magnitudes brighter than the detection limit in that galaxy. The control time contributed to the search by that observation will be unbiased by any initial assumptions, and simply be the time since the previous observation. In contrast, a point in the upper right of the diagram represents an image taken many months after the last observation of the field, with some supernovae types not detectable even if they had occurred a few days beforehand. The control time contributed to the total by this observation is given by the visibility duration of the various supernova types for that limiting magnitude, and this can cause bias in the result. Note that some overlap may exist-for example, 50 observations of the same galaxy, each exactly 10 days apart, would show up as only one dot, with an $\mathrm{X}$ coordinate of 10 days.

Also shown as large dots are the five supernovae detected during the search. The large dot indicates date of discovery, with the bars from some events indicating the time of maximum light, where it is appreciably before the discovery date. The magnitudes used here are the detection limits for the discovery images, not the $\mathrm{R}$ magnitudes of the supernova candidates at time of detection. 
The limited number of discoveries so far leads to large uncertainties for our estimated rates. We can loosely say that the Type $\mathrm{Ib} / \mathrm{c}$ rate is approximately $0.43 \mathrm{SNu}(+0 \cdot 11,-0 \cdot 08$, depending on the control time), plus a Poisson $\sqrt{N}$ error of around $\pm 95 \%$ due to the small number of discoveries. Similarly, we can determine that the Type IIP rate is around $0 \cdot 86 \mathrm{SNu}$ $(+0 \cdot 18,-0 \cdot 14)$, plus a Poisson $\sqrt{N}$ error of $\pm 54 \%$. Adding the errors in quadrature, we get $0.43 \pm 0.43$ $\mathrm{SNu}$ for Type Ib/c events, and $0 \cdot 86 \pm 0.49 \mathrm{SNu}$ for Type IIP events. Adding the errors in quadrature is not formally correct, and the errors are also not symmetric about the expectation values given, since the rates must obviously be positive, but they are only intended to give a reasonable estimate for the expected variation. Here $\mathrm{SNu}$ are 'supernova units', expressed in supernovae per $10^{10} \mathrm{~L}_{\mathrm{B}_{\odot}}$ galaxy per century. These rates are for a Hubble constant of $75 \mathrm{~km} \mathrm{~s}^{-1} \mathrm{Mpc}^{-1}$, and scale as $\left(H_{0} / 75\right)^{2}$.

Our values are in reasonable agreement with other published results. Cappellaro et al. (1993) gave a Ia rate of $0 \cdot 39 \pm 0 \cdot 19 \mathrm{SNu}, \mathrm{a} \mathrm{Ib} / \mathrm{c}$ rate of $0 \cdot 27 \pm 0 \cdot 18$, and a combined Type II rate of $1 \cdot 48 \pm 0 \cdot 65 \mathrm{SNu}$. The errors given for these include terms for estimated errors in light curve and sensitivity parameters, and errors in determining inclination corrections.

\section{Conclusions}

Although the rates derived here for the first three years of operation have a high uncertainty due to small-number statistics, the systematic errors present are expected to be fairly small, due to the lack of control time dependence on visibility time. Perth Observatory will be installing automatic weather sensors for the two automatic telescopes on site, within the next 12 months. These, together with a dewar upgrade for the CCD camera to improve hold-time, will allow the search to run more often, and should double or triple the supernova discovery rate. In addition to finding more nearby supernovae near maximum light for further study, this will allow us to improve our SN rate precision.

\section{Acknowledgments}

This research has made use of the NASA/IPAC Extragalactic Database (NED) which is operated by the Jet Propulsion Laboratory, Cal'Tech, under contract with the National Aeronautics and Space Administration. We also thank the Astronomical Data Center (ADC) and the National Space Science Data Center through the World Data Center A for Rockets and Satellites, for providing catalogue data on the ADC 'Selected Astronomical Catalogs' CD-ROM.

Note added in proof: A sixth confirmed supernova has recently been discovered by the search systemSN1997Z in NGC 3261, published in IAUC 6558.

Blanton, E. L., Schmidt, B. P., Kirshner, R. P., Ford, C. H., Chromey, F. R., \& Herbst, W. 1995, AJ, 110, 2868

Branch, D., Livio, M., Yungelson, L. R., Boffi, F. R., \& Baron, E. 1995, PASP, 107, 1019

Cappellaro, E., Turatto, M., Benetti, S., Tsvetkov, D. Yu., Bartunov, O. S., \& Makarova, I. N. 1993, A\&A, 273, 383

Eastman, R. G., Woosley, S. E., Weaver, T. A., \& Pinto, P. A. 1994, ApJ, 430, 300

Evans, R. 1995, IAUC 6239

Gunn, J. E., Emory, E. B., Harris, F. H., \& Oke, J. B. 1987, PASP, 99, 518

Martin, R., \& Williams, A. J. 1994, IAUC 6003

Muller, R. A., Newburg, H. J. M., Pennypacker, C. R., Perlmutter, S., Sasseen, T. P., \& Smith, C. K. 1992 ApJ, 384, L9

Richmond, M. W. 1992, Ph.D. Dissertation (U. of California, Berkeley) (see also http://www.astro.princeton.edu/ richmond/diss)

Tammann, G. A., Löffler, W., \& Schröder, A. 1994, ApJS, 92,487

Williams, A. J., \& Martin, R. 1993, IAUC 5733

Williams, A. J., \& Martin, R. 1994, IAUC 6120

Williams, A. J., \& Martin, R. 1995, IAUC 6206

Williams, A. J., \& Martin, R. 1996, IAUC 6351

Williams, A. J., Martin, R., Verveer, A., Blair, D. G., Koch, R., Lynch, M. J., \& Burman, R. 1995, Rev. Sci. Instrum., 66,2777

Woosley, S. E., Langer, N., \& Weaver, T. A. 1993, ApJ, 411,823

Woosley, S. E., Langer, N., \& Weaver, T. A. 1995, ApJ, 448,315 